\title{
Genetic Structure of Cartagena de Indias Population Using Hypervariable Markers of Y Chromosome
}

\author{
Doris Esther Gómez-Camargo ${ }^{1^{*}}$, Rafael Camacho-Mejorado ${ }^{2 *}$, Claudio Gómez-Alegría ${ }^{3}$, \\ Angelo Alario", Esther Alhelí Henández-Tobías ${ }^{2}$, Gustavo Mora-García ${ }^{1}$, \\ Marco Antonio Meraz-Ríos ${ }^{5}$, Rocío Gómez ${ }^{* \#}$ \\ ${ }^{1}$ Doctorado en Medicina Tropical, Facultad de Medicina, Universidad de Cartagena, Cartagena de Indias, \\ Colombia \\ ${ }^{2}$ Departamento de Toxicología, Cinvestav-IPN, México D.F., México \\ ${ }^{3}$ Departamento de Farmacia, Facultad de Ciencias, Universidad Nacional de Colombia, Bogotá, Colombia \\ ${ }^{4}$ Departamento Médico, Facultad de Medicina, Universidad de Cartagena, Cartagena de Indias, Colombia \\ ${ }^{5}$ Departamento de Biomedicina Molecular, Cinvestav-IPN, México D.F., México \\ Email:" $\underline{\text { mrgomez@cinvestav.mx }}$
}

Received 9 March 2015; accepted 24 March 2014; published 30 March 2015

Copyright (C) 2015 by authors and Scientific Research Publishing Inc.

This work is licensed under the Creative Commons Attribution International License (CC BY).

http://creativecommons.org/licenses/by/4.0/

c) (i) Open Access

\section{Abstract}

Ethnicity has been associated with the incidence of diseases and consequently it is a cornerstone in medical genetic studies. It is mainly important in admixture populations, where the population stratification can produce spurious results that lead to erroneous conclusions. Consequently, population stratification has become one of the most important confounding factors in populationbased genetic association studies, especially in Latino populations. Cartagena de Indias is a cosmopolitan city with dissimilar ancestry proportions due to recent miscegenation. This population mainly exhibits African and Amerindian matrilineal ancestries. Nevertheless, important asymmetries in the paternal genetic history related to the complex patterns of migration in the colonial period increase the male genetic diversity in this population. As a result of this recent admixture, population stratification has arisen, where each subpopulation is not equally represented. Consequently, the allele differences between cases and controls could be related with different frequencies among different population strata rather than the association of the genes with the disease. In order to define the patrilineal substructure of the Cartagena's population, a total of 130 unrelated men were ancestrally studied using 15 Y-STR loci routinely employed in anthropological, forensic and population genetics. Our results show that Cartagena is an admixture population consisting of European (80\%), Amerindian (10\%) and African ancestries (10\%), which are

\footnotetext{
*These authors equally contributed to this work.

${ }^{\#}$ Corresponding author.
}

How to cite this paper: Gómez-Camargo, D.E., Camacho-Mejorado, R., Gómez-Alegría, C., Alario, A., Henández-Tobías, E.A., Mora-García, G., Meraz-Ríos, M.A. and Gómez, R. (2015) Genetic Structure of Cartagena de Indias Population Using Hypervariable Markers of Y Chromosome. Open Journal of Genetics, 5, 27-41. http://dx.doi.org/10.4236/ojgen.2015.51003 
represented by haplogroups R1b and I2a (XI2a1), Q-M242/Q-M3, and E1b1a/E1b1b, respectively. Complex genetic patterns found in Cartagena's population emphasize the importance to know the genetic variation in order to diminish the inconsistence for future genetic association studies. In addition, our findings illustrate the complex genetic background of Cartagena population and reinforce the need to encompass more geographic regions to generate more robust data for anthropological and forensic applications.

\section{Keywords}

Population Genetics, Y-Chromosome, Microsatellites, Cartagena, Colombia

\section{Introduction}

Cartagena de Indias is a city in the north of Colombia located on the shores of the Caribbean Sea and is the capital of the Department of Bolivar. Cartagena is one of the oldest cities on the Colombian Caribbean coast founded in 1533 by Spanish conquerors [1]. This city also represented the primary slaving port during the early colonial period (1570-1640), where more than 150,000 Africans coming from western Africa (Angola and the Guineas, principally) were introduced into Americas [2]. As a consequence, the ethnic composition of Cartagena is the result of a three-hybrid fusion: the native aborigines known as the Calamari; the European conquerors (principally from Spain); and the African slaves [3]. This ancestral admixture has predominantly resulted in a mulatto, mestizo, Amerindian, and Afro-descendant population. However, recent evidences show a disproportionate contribution of European males and Amerindian females, provoking a sex bias in admixture proportions [4] [5].

Genetic association studies are a powerful strategy for identifying genotypic-phenotypic associations in complex diseases. Recent findings confirm that populations with multiple ethnic origins show important differences in allelic and genotypic frequencies, which may inflate false positive rates causing a spurious association because the genetic stratification is unevenly distributed across different subpopulations [6]. Therefore, allele differences between cases and controls could be related with systematic differences in ancestry as well as dissimilar frequencies among different population strata rather than a real association of the genes with the disease [7] [8]. These differences are more important in young populations with complex patterns of admixture such as Latin American populations [9]. Hence, population stratification is the principal confounder variable on genetic association studies, causing bias that may yield misleading results, which could be used in the practice of medicine and public health [10].

Focused on identifying the patrilineal contribution in Cartagena's population and to avoid spurious associations, we determined the genetic structure of Cartagena de Indias population using Y-Chromosome Short Tandem Repeats (Y-STR). We analyzed 130 unrelated men, using 15 Y-STR loci which is routinely employed in human migration and evolutionary studies as well as genetic structure and forensic analysis among others [11] [12]. Our findings show that Cartagena's population is highly diverse, showing patrilineal ancestry from European, Middle Eastern, African and Amerindian populations. These findings highlight the importance of knowing local-specific patterns throughout the country in order to establish the population stratification and correct the impact of admixture in future genetic association studies. In addition, genetic variability studies provide information about population history, as well as the relationship between some diseases and ancestral lineages, which could also be used in the subsequent epidemiological studies [13].

\section{Materials and Methods}

\subsection{Population}

We analyzed the STR genetic data of 130 unrelated individuals born in Cartagena de Indias, Colombia with at least three generations of ancestors who had been born in this city (Figure 1). The questionnaire for choosing candidates was designed to ensure that the participants were not filially related, i.e., no one was son or brother of another. This study was approved by the Ethics Research Committee of the University of Cartagena, Colombia (Resolution Number 46, 2012). Each individual signed an informed consent to participate in this study. 


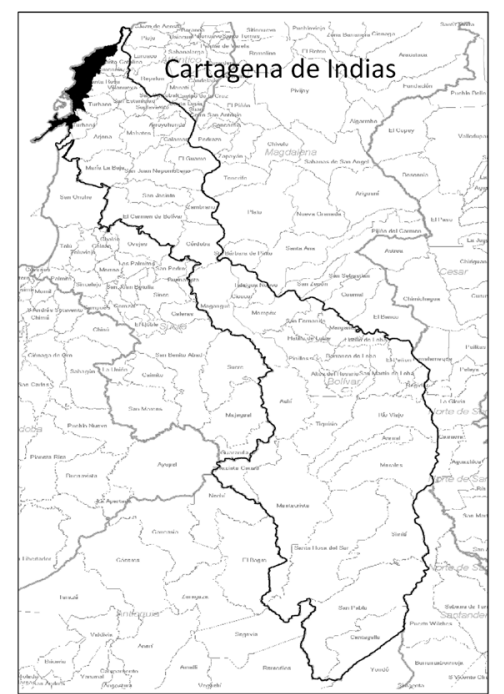

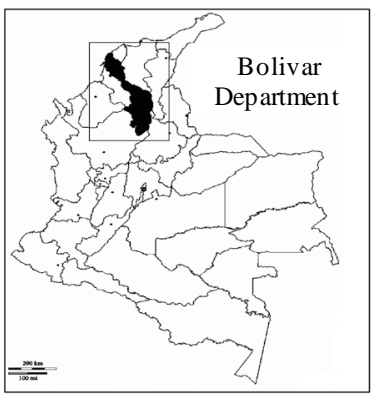

Republic of Colombia

Figure 1. Map of the republic of Colombia showing Bolivar Department and the location of cartagena de Indias.

\subsection{Molecular Analysis}

Genomic DNA was extracted from peripheral blood leukocytes using Qiamp DNA mini kit (Qiagen, Düsseldorf, Germany). Approximately $10 \mathrm{ng}$ target DNA was amplified using fifteen Y-chromosome short tandem repeat markers described previously (Table 1). Amplicons were obtained using multiplex reactions described in Table 2. The resulting amplicons were carried out on the ABI Prism 3130XL Genetic Analyzer using GeneMapper ID v.3.2. software (Applied Biosystems, Carlsbad, CA, USA).

\subsection{Quality Control}

Control DNA 007 was used as international validated internal controls (Applied Biosystems, Carlsbad, CA, USA).

\subsection{Statistical Analysis}

Allelic and haplotypic frequencies, number of alleles (k), haplotype diversity (HD), genetic diversity over loci $(h)$, and mean pairwise differences (M) were estimated using Arlequin software v 3.5 [14]. The number of unique haplotypes (UH) was estimated by direct counting. In order to compare our data with other populations, haplotype and haplogroup information was collected from previous reports. A total of 1372 individuals from different Colombian populations were included in the database and used for further analysis [15]-[17]. Y haplogroups were determined from Y-STR haplotypes with haplogroup predictor software (http://www.hprg.com/hapest5/), using equal priority to estimate the probability of assignment to a particular haplogroup [18]. The phylogenetic relationship of STR haplotypes was analyzed with Network v 4.6.1.1 software [19], which was built with Network Publisher software using a median joining approach and MP post-processing. Each haplotype was connected to all other haplotypes from which it differed by one repeat unit step at a single microsatellite locus. The Y-STR loci were weighted based on the inverse of their variances.

\section{Results}

\subsection{Y-Chromosome STR Diversity}

The haplotype distribution of the 15 Y-STR loci in the 130 individuals studied is shown in Table 3 . The distribution of allele frequencies, number of different alleles $(k)$, and locus diversity $(h)$ are shown in Table 4 . The most diverse loci were DYS458 $(h=0.775)$, DYS438/DYS390 $(h=0.766)$, DYS635/DYS389II $(h=0.740)$, and DYS19 $(h=0.721)$; while DYS393 $(h=0.406)$ was the least diverse. 
Table 1. Primer sequences used for the multiplex reactions.

\begin{tabular}{|c|c|c|c|c|}
\hline Uorochrome & \multicolumn{2}{|c|}{ STR Locus } & Primer Sequence & Primer Seq. Ref. \\
\hline \multirow{2}{*}{ PET } & \multirow{2}{*}{ DYS19 } & $\mathrm{F}$ & ACTACTGAGTTTCTGTTATAGTGTTTTT & \multirow{2}{*}[11]{} \\
\hline & & $\mathrm{R}$ & GTCAATCTCTGCACCTGGAAAT & \\
\hline \multirow{2}{*}{ VIC } & \multirow{2}{*}{ DYS389I } & $\mathrm{F}$ & ССААСТСТСАТСТGTATTATCTATG & \multirow{2}{*}{ [11] } \\
\hline & & $\mathrm{R}$ & GTTATCCCTGAGTAGTAGAAGAATG & \\
\hline \multirow{2}{*}{ VIC } & \multirow{2}{*}{ DYS389II } & $\mathrm{F}$ & CСAACTCTCATCTGTATTATCTATG & \multirow{2}{*}{ [11] } \\
\hline & & $\mathrm{R}$ & GTTATCCCTGAGTAGTAGAAGAATG & \\
\hline \multirow{2}{*}{ VIC } & \multirow{2}{*}{ DYS390 } & $\mathrm{F}$ & TATATTTTACACATTTTTGGGCCC & \multirow{2}{*}{ [40] } \\
\hline & & $\mathrm{R}$ & GTGACAGTAAAATGAAAACATTGC & \\
\hline \multirow{2}{*}{ 6-FAM } & \multirow{2}{*}{ DYS391 } & $\mathrm{F}$ & TTCAATCATACACCСАTATCTGTC & \multirow{2}{*}{ [11] } \\
\hline & & $\mathrm{R}$ & GATAGAGGGATAGGTAGGCAGGC & \\
\hline \multirow{2}{*}{ NED } & \multirow{2}{*}{ DYS392 } & $\mathrm{F}$ & TAGAGGCAGTCATCGCAGTG & \multirow{2}{*}{ [40] } \\
\hline & & $\mathrm{R}$ & GACCTACCAATCCСАТТССТT & \\
\hline \multirow{2}{*}{ VIC } & \multirow{2}{*}{ DYS393 } & $\mathrm{F}$ & GTGGTCTTCTACTTGTGTCAATAC & \multirow{2}{*}[11]{} \\
\hline & & $\mathrm{R}$ & GAACTCAAGTCCAAAAAATGAGG & \\
\hline \multirow{2}{*}{ 6-FAM } & \multirow{2}{*}{ DYS437 } & $\mathrm{F}$ & GACTATGGGCGTGAGTGCAT & \multirow{2}{*}[40]{} \\
\hline & & $\mathrm{R}$ & GAGACCCTGTCATTCACAGATGA & \\
\hline \multirow{2}{*}{ VIC } & \multirow{2}{*}{ DYS438 } & $\mathrm{F}$ & CCAAAATTAGTGGGGAATAGTTG & \multirow{2}{*}{ [11] } \\
\hline & & $\mathrm{R}$ & GATCACCCAGGGTCTGGAGTT & \\
\hline \multirow{2}{*}{ NED } & \multirow{2}{*}{ DYS439 } & $\mathrm{F}$ & TCGAGTTGTTATGGTTTTAGGTCT & \multirow{2}{*}{ [11] } \\
\hline & & $\mathrm{R}$ & GTGGCTTGGAATTCTTTTACCC & \\
\hline PET & DYS448 & $\mathrm{F}$ & TGGGAGAGGCAAGGATCCAA & [11] \\
\hline 101 & $014+40$ & $\mathrm{R}$ & GTCATATTTCTGGCCGGTCTGG & {$[11]$} \\
\hline $\mathrm{VIC}$ & DYS456 & $\mathrm{F}$ & GGACCTTGTGATAATGTAAGATAG & [111] \\
\hline VIC & DYS456 & $\mathrm{R}$ & GTAGAGGGACAGAACTAATGGAA & [41] \\
\hline 6-FAM & DYS458 & $\mathrm{F}$ & GCAACAGGAATGAAACTCCAAT & [111] \\
\hline 6-FAM & DYS458 & $\mathrm{R}$ & GTTCTGGCATTACAAGCATGAG & [11] \\
\hline NFD & DYS635 & $\mathrm{F}$ & ACCAGCCСAАATATCCATCA & [42] \\
\hline NED & DYSO35 & $\mathrm{R}$ & TGGAATGCTCTCTTGGCTTC & {$[42]$} \\
\hline 6-FAM & $\mathrm{Y}-\mathrm{CATA}-\mathrm{H} 4$ & $\mathrm{~F}$ & GAGACCTAAGCAGAGATGTTGGTTTTC & {$[\Delta 11]$} \\
\hline & & $\mathrm{R}$ & CCTCTGATGGTGAAGTAATGGAATTAGA & \\
\hline
\end{tabular}

Table 2. Cartagena amplification conditions for PCR multiplex of tested loci.

\begin{tabular}{|c|c|c|c|c|c|c|c|c|}
\hline & STR locus & Primers [ ] $(\mu \mathbf{M})$ & Reactive & Final [ ] & PCR conditions & Temp ${ }^{\circ} \mathrm{C}$ & Time & Cycles \\
\hline \multirow{5}{*}{ 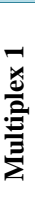 } & DYS391 & 0.8 & Buffer & $1 \mathrm{X}$ & Pre-denaturalization & 95 & $10 \mathrm{~min}$ & \multirow{5}{*}{30} \\
\hline & DYS437 & 0.7 & $\mathrm{MgCl}_{2}$ & $1.75 \mathrm{mM}$ & Denaturalization & 94 & $1 \mathrm{~min}$ & \\
\hline & DYS439 & 0.048 & dNTP & $200 \mathrm{mM}$ & Annealing & 55 & $1 \mathrm{~min}$ & \\
\hline & DYS448 & 0.016 & Betaina & $0.83 \mathrm{M}$ & Extension & 70 & $1 \mathrm{~min}$ & \\
\hline & DYS458 & 0.9 & TaqPol & $1 \mathrm{U}$ & Post-extension & 60 & $45 \min$ & \\
\hline \multirow{5}{*}{$\frac{N}{\stackrel{x}{d}}$} & DYS19 & 1.2 & Buffer & $1 \mathrm{X}$ & Pre-denaturalization & 95 & $10 \mathrm{~min}$ & \multirow{5}{*}{30} \\
\hline & DYS393 & 0.2 & $\mathrm{MgCl}_{2}$ & $1.75 \mathrm{mM}$ & Denaturalization & 94 & $1 \mathrm{~min}$ & \\
\hline & DYS438 & 0.25 & dNTP & $100 \mathrm{mM}$ & Annealing & 55 & $1 \mathrm{~min}$ & \\
\hline & GATA-H4 & 0.4 & Betaina & $0.83 \mathrm{M}$ & Extension & 70 & $1 \mathrm{~min}$ & \\
\hline & & & TaqPol & $1 \mathrm{U}$ & Post-extension & 60 & $45 \mathrm{~min}$ & \\
\hline \multirow{6}{*}{$\frac{n}{\stackrel{a}{a}}$} & DYS389I & 0.25 & Buffer & $1 \mathrm{X}$ & Pre-denaturalization & 95 & $10 \mathrm{~min}$ & \multirow{6}{*}{30} \\
\hline & DYS389II & 0.25 & $\mathrm{MgCl}_{2}$ & $1.75 \mathrm{mM}$ & Denaturalization & 94 & $1 \mathrm{~min}$ & \\
\hline & DYS390 & 0.2 & dNTP & $100 \mathrm{mM}$ & Annealing & 55 & $1 \mathrm{~min}$ & \\
\hline & DYS392 & 0.5 & Betaina & $0.83 \mathrm{M}$ & Extension & 70 & $1 \mathrm{~min}$ & \\
\hline & DYS456 & 0.2 & TaqPol & $1 \mathrm{U}$ & Post-extension & 60 & $45 \mathrm{~min}$ & \\
\hline & DYS635 & 0.5 & & & & & & \\
\hline
\end{tabular}


Table 3. Cartagena de Indias haplogroup identification from haplotype definition, with fitness score and probability.

\begin{tabular}{|c|c|c|c|c|c|c|c|c|c|c|c|c|c|c|c|c|c|c|}
\hline 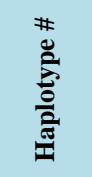 & $\begin{array}{l}\vec{D} \\
\text { त्रे }\end{array}$ & 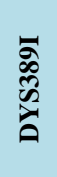 & 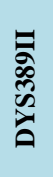 & 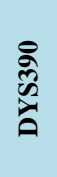 & $\begin{array}{l}\overline{\mathscr{D}} \\
\text { त्रे } \\
\text { ద }\end{array}$ & 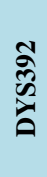 & $\begin{array}{l}\mathscr{P} \\
\text { क्रे } \\
\text { ద }\end{array}$ & \begin{tabular}{l}
$\hat{0}$ \\
\multirow{3}{*}{} \\
$\hat{n}$
\end{tabular} & $\begin{array}{l}\infty \\
\text { స్ } \\
\text { ప્ર }\end{array}$ & 蔦 & 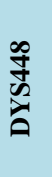 & 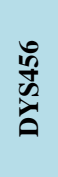 & 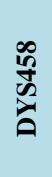 & 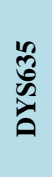 & 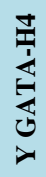 & 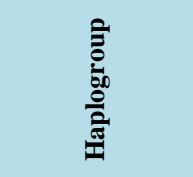 & 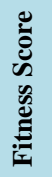 & 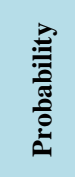 \\
\hline hc-16 & 14 & 13 & 29 & 24 & 11 & 12 & 15 & 17 & 10 & - & 20 & 18 & 17 & 21 & 12 & $\mathrm{I} 2 \mathrm{~b} 1$ & 16 & 0.682 \\
\hline hc-23 & 14 & 13 & 28 & 25 & 11 & 12 & 12 & 17 & 10 & 12 & 19 & 14 & 16 & 21 & 11 & $\mathrm{~L}$ & 27 & 0.778 \\
\hline hc-24 & 13 & 14 & 30 & 25 & 11 & 14 & 13 & 16 & 13 & 12 & 19 & 16 & 17 & 23 & 12 & $\mathrm{R} 1 \mathrm{~b}$ & 31 & 0.981 \\
\hline hc-30 & 15 & 14 & 28 & 24 & 11 & 12 & 13 & 15 & 10 & 13 & 21 & 15 & 17 & 22 & 12 & I2a1 & 34 & 0.864 \\
\hline hc-33 & 13 & 13 & 29 & 23 & 11 & 12 & 12 & 18 & 10 & 12 & 20 & 16 & 14 & 19 & 11 & $\mathrm{~L}$ & 14 & 0.623 \\
\hline hc- 40 & 14 & 13 & 29 & 24 & 11 & 12 & 13 & 17 & 10 & 13 & 20 & 16 & 19 & 21 & 11 & E1b1b & 25 & 0.503 \\
\hline hc-42 & - & 14 & 30 & 22 & - & 12 & - & - & - & - & 20 & 17 & - & 21 & - & $\mathrm{I} 2 \mathrm{~b} 1$ & 54 & 0.95 \\
\hline hc-67 & - & 14 & 29 & 25 & - & 14 & - & - & - & 13 & 19 & 16 & - & 23 & - & R1b & 33 & 0.843 \\
\hline hc-89 & 15 & 15 & 31 & 24 & 11 & 13 & 14 & 15 & 10 & 11 & 20 & 16 & 15 & 22 & 11 & $\mathrm{I} 2 \mathrm{~b} 1$ & 31 & 0.936 \\
\hline hc-95 & 14 & 13 & 29 & 29 & 11 & 13 & 13 & 15 & 12 & 12 & 19 & 16 & 16 & 23 & 12 & $\mathrm{R} 1 \mathrm{~b}$ & 33 & 1 \\
\hline hc-111 & - & 14 & 29 & 25 & 12 & 14 & 13 & 16 & - & 12 & 19 & 17 & 16 & 23 & - & R1b & 28 & 0.995 \\
\hline hc-113 & 15 & 15 & 32 & 23 & 11 & 12 & 13 & 15 & 11 & 12 & 21 & 16 & 16 & 21 & 10 & E1b1a & 32 & 0.771 \\
\hline hc-115 & 13 & 14 & 30 & 25 & 12 & 14 & 12 & 16 & 11 & 13 & 19 & 16 & 16 & 23 & 13 & $\mathrm{Q}$ & 20 & 0.687 \\
\hline hc-120 & 14 & 14 & 30 & 23 & 11 & 12 & 14 & 17 & 11 & 11 & 20 & 17 & 17 & 21 & 11 & $\mathrm{I} 2 \mathrm{~b} 1$ & 23 & 0.916 \\
\hline hc-142 & 13 & 14 & 29 & 25 & 11 & 14 & 14 & 16 & 13 & 12 & 19 & 16 & 19 & 23 & 12 & $\mathrm{R} 1 \mathrm{~b}$ & 18 & 0.879 \\
\hline hc-149 & 14 & 14 & 29 & 23 & 11 & 14 & 13 & 16 & 13 & 11 & 19 & 16 & 18 & 23 & 12 & $\mathrm{R} 1 \mathrm{~b}$ & 31 & 1 \\
\hline hc-159 & - & 14 & 30 & 25 & 11 & 14 & - & 16 & - & - & 19 & 16 & 17 & 23 & - & $\mathrm{R} 1 \mathrm{~b}$ & 44 & 0.993 \\
\hline hc-161 & 13 & 16 & 31 & 24 & 11 & 12 & 12 & 15 & 9 & 11 & 20 & 17 & 15 & 22 & 11 & J2a1 x J2a1-bh & 19 & 0.906 \\
\hline hc-162 & - & 13 & - & 25 & 12 & 14 & - & 16 & - & 13 & 19 & 16 & 16 & 23 & - & $\mathrm{R} 1 \mathrm{~b}$ & 31 & 0.991 \\
\hline hc-165 & - & 14 & 31 & 25 & 10 & 12 & - & 15 & - & 12 & 20 & 16 & 16 & 23 & - & E1b1b & 42 & 0.388 \\
\hline hc-168 & 13 & 15 & 30 & 25 & 13 & 14 & 13 & 16 & 13 & 11 & 19 & 16 & 18 & 23 & 11 & $\mathrm{R} 1 \mathrm{~b}$ & 14 & 1 \\
\hline hc-169 & 14 & 13 & 28 & 25 & 11 & 12 & 12 & 17 & - & 12 & - & 14 & 16 & 21 & 11 & $\mathrm{~J} 2 \mathrm{~b}$ & 17 & 0.843 \\
\hline hc-170 & - & 14 & 29 & 25 & 11 & 14 & - & 15 & - & - & 18 & 15 & 18 & 24 & - & $\mathrm{R} 1 \mathrm{~b}$ & 30 & 0.979 \\
\hline hc-172 & - & 14 & 30 & 22 & 11 & 12 & - & 15 & - & 11 & 21 & 16 & - & 21 & - & J2a1 x J2a1-bh & 38 & 0.353 \\
\hline hc-179 & - & 13 & 29 & 23 & 11 & 12 & - & 15 & - & - & - & 16 & 16 & 22 & - & I2b1 & 47 & 0.462 \\
\hline hc-184 & - & 15 & - & 24 & 11 & 15 & 13 & 15 & - & 13 & 19 & 17 & 15 & 22 & 11 & $\mathrm{Q}$ & 35 & 0.983 \\
\hline hc-185 & - & 14 & 30 & 25 & 11 & 14 & - & 16 & - & 12 & - & 16 & 17 & 23 & - & $\mathrm{R} 1 \mathrm{~b}$ & 44 & 0.997 \\
\hline hc-186 & - & - & - & 23 & 11 & 12 & 13 & 15 & - & 13 & 21 & 16 & 18 & 22 & - & I2a (xI2a1) & 40 & 0.522 \\
\hline hc-191 & 14 & 13 & 29 & 23 & 11 & 13 & 13 & 15 & 12 & 13 & 19 & 17 & 17 & 23 & 13 & $\mathrm{R} 1 \mathrm{~b}$ & 58 & 1 \\
\hline hc-200 & - & 15 & 31 & 22 & 11 & 12 & 15 & 15 & - & 12 & 21 & 17 & 17 & 21 & 10 & I2b1 & 24 & 0.841 \\
\hline hc-202 & - & 13 & 28 & 25 & 11 & 12 & - & 17 & - & 12 & - & 14 & 17 & 22 & - & I2a (xI2a1) & 23 & 0.688 \\
\hline hc-204 & 15 & 14 & 30 & 22 & 11 & 12 & 13 & 15 & 11 & 12 & 22 & 15 & 15 & 21 & 13 & J2a1 x J2a1-bh & 23 & 0.68 \\
\hline hc-219 & - & 13 & - & 25 & 11 & 14 & - & 15 & - & 12 & 18 & 19 & 17 & 23 & - & R1b & 36 & 0.991 \\
\hline
\end{tabular}




\section{Continued}

\begin{tabular}{|c|c|c|c|c|c|c|c|c|c|c|c|c|c|c|c|c|c|c|}
\hline hc-221 & 12 & 14 & 29 & 24 & 11 & 15 & 12 & 16 & 11 & - & - & 17 & 17 & 23 & 12 & $\mathrm{Q}$ & 16 & 0.534 \\
\hline hc-223 & - & 15 & 31 & 23 & - & - & - & - & - & - & - & 16 & - & 21 & - & $\mathrm{T}$ & 51 & 0.464 \\
\hline hc-230 & - & - & - & - & 11 & - & - & 16 & - & - & 20 & - & 15 & - & - & I1 & 57 & 0.873 \\
\hline hc-231 & 14 & 14 & - & 22 & 13 & - & 13 & 15 & 11 & 11 & 21 & 16 & 16 & 21 & 12 & E1b1a & 19 & 0.845 \\
\hline hc-232 & 13 & 14 & 31 & 25 & 13 & 14 & 13 & 15 & - & 12 & 19 & 17 & 15 & 23 & - & R1b & 20 & 0.96 \\
\hline hc-233 & 13 & 14 & 29 & 25 & 11 & 14 & 13 & 16 & 13 & 12 & 19 & 16 & 17 & 24 & 12 & R1b & 23 & 0.974 \\
\hline hc-235 & 13 & 14 & 29 & 25 & 11 & 14 & 13 & 16 & 13 & 12 & 19 & 17 & 17 & 23 & 12 & $\mathrm{R} 1 \mathrm{~b}$ & 25 & 0.958 \\
\hline hc-238 & 15 & 14 & 30 & 26 & 11 & 12 & 13 & 15 & 11 & 11 & 20 & 18 & 15 & 23 & 13 & R1a & 30 & 1 \\
\hline hc-240 & 13 & 14 & 29 & 26 & 12 & 14 & 14 & 16 & 13 & 12 & 19 & 18 & 19 & 24 & 12 & R1b & 9 & 0.827 \\
\hline hc-251 & - & 14 & 28 & 25 & 12 & 14 & - & 16 & - & 12 & - & 17 & 17 & 23 & - & $\mathrm{R} 1 \mathrm{~b}$ & 17 & 0.987 \\
\hline hc-254 & 13 & 15 & - & 24 & 13 & 15 & 13 & 15 & - & 13 & 20 & 17 & 16 & 23 & 12 & R1b & 11 & 0.914 \\
\hline hc-255 & 13 & - & - & - & 11 & - & 13 & 16 & 9 & 11 & 22 & - & 16 & - & 11 & $\mathrm{~J} 2 \mathrm{~b}$ & 15 & 0.357 \\
\hline hc-266 & 13 & 13 & 29 & 25 & 11 & 12 & 13 & 15 & 10 & 11 & 20 & 16 & 14 & 21 & 11 & E1b1b & 36 & 0.969 \\
\hline hc-268 & 16 & 14 & 30 & 22 & 11 & 12 & 14 & 15 & 11 & 13 & 21 & 17 & 16 & 21 & 11 & E1b1a & 27 & 0.573 \\
\hline hc-269 & 12 & 14 & 30 & 25 & 11 & 14 & 13 & 15 & 10 & 13 & 21 & 17 & 15 & 23 & 12 & $\mathrm{Q}$ & 20 & 0.945 \\
\hline hc-271 & 13 & 14 & 29 & 26 & 11 & 15 & 13 & 16 & 13 & 13 & 19 & 16 & 16 & 23 & 12 & $\mathrm{R} 1 \mathrm{~b}$ & 15 & 0.829 \\
\hline hc-274 & 13 & 14 & 29 & 26 & 12 & 14 & 13 & 16 & 13 & 12 & 19 & 16 & 17 & 23 & 12 & $\mathrm{R} 1 \mathrm{~b}$ & 17 & 0.99 \\
\hline hc-278 & 14 & 13 & 29 & 24 & 11 & 13 & 13 & 15 & 10 & 13 & 19 & 16 & 17 & 23 & 12 & $\mathrm{R} 1 \mathrm{~b}$ & 53 & 1 \\
\hline hc-280 & - & 13 & 30 & 22 & 11 & 12 & - & 15 & - & 12 & - & 16 & 18 & 21 & - & J2a1b & 44 & 0.796 \\
\hline hc-284 & - & - & - & - & 13 & - & 13 & 16 & - & 12 & 20 & - & 17 & - & - & I2a (xI2a1) & 24 & 0.791 \\
\hline hc-285 & 14 & - & - & - & 13 & - & 13 & 18 & 8 & 11 & - & - & 17 & - & 9 & I2b (xI2b1) & 8 & 0.993 \\
\hline hc-288 & - & 14 & 30 & 22 & 11 & 13 & - & 15 & - & 12 & - & 16 & 19 & 21 & - & $\mathrm{T}$ & 45 & 0.519 \\
\hline hc-291 & 12 & 15 & 30 & 24 & 13 & 14 & 13 & 15 & 13 & 13 & 18 & 17 & 17 & 23 & 11 & $\mathrm{R} 1 \mathrm{~b}$ & 14 & 1 \\
\hline hc-300 & - & 15 & - & 25 & 13 & 14 & - & 16 & - & - & - & 16 & 17 & 24 & - & R1b & 9 & 0.98 \\
\hline hc-308 & 14 & 14 & 29 & 24 & 11 & 15 & 13 & 15 & 9 & 12 & 19 & 15 & 17 & 21 & 11 & $\mathrm{~T}$ & 51 & 1 \\
\hline hc-309 & - & 15 & - & 24 & 11 & 15 & - & 15 & - & - & 19 & 15 & 17 & 21 & - & $\mathrm{T}$ & 32 & 0.692 \\
\hline hc-310 & 12 & 14 & 31 & 25 & 11 & 13 & 13 & 15 & 10 & 11 & 21 & 18 & 17 & 23 & 12 & $\mathrm{R} 1 \mathrm{~b}$ & 14 & 0.484 \\
\hline hc-314 & - & 15 & 30 & 26 & 12 & 15 & - & 16 & - & 11 & - & 17 & 17 & 23 & - & $\mathrm{R} 1 \mathrm{~b}$ & 9 & 0.979 \\
\hline hc-315 & 14 & 14 & 29 & 24 & 11 & 15 & 13 & 15 & 9 & 12 & 19 & 15 & 17 & 21 & 11 & $\mathrm{~T}$ & 51 & 1 \\
\hline hc-317 & - & 14 & 30 & 25 & 11 & 14 & - & 14 & - & - & - & 17 & 15 & 22 & - & $\mathrm{Q}$ & 48 & 0.646 \\
\hline hc-347 & - & 14 & 28 & 24 & 11 & 12 & 13 & 16 & - & 11 & 21 & 15 & 15 & 24 & - & I2a (xI2a1) & 24 & 0.899 \\
\hline hc-350 & - & 14 & 28 & 24 & 11 & - & - & 16 & - & - & - & 15 & 15 & 24 & - & $\mathrm{L}$ & 28 & 0.747 \\
\hline hc-356 & 17 & 13 & 28 & 23 & 10 & 11 & 13 & 15 & 10 & 11 & 21 & 14 & 15 & 24 & 12 & I2a1 & 66 & 1 \\
\hline hc-358 & - & 14 & 28 & 24 & 11 & 12 & - & 15 & - & 11 & 21 & 15 & 15 & 24 & - & I2a (xI2a1) & 29 & 0.517 \\
\hline hc-360 & 15 & 13 & 32 & 21 & 10 & 11 & 13 & 14 & 11 & 11 & 20 & 15 & 16 & 21 & 12 & E1b1a & 66 & 1,00 \\
\hline hc-362 & 14 & 13 & 29 & 23 & 10 & 10 & 15 & 16 & 11 & 11 & 19 & 15 & 17 & 25 & 12 & $\mathrm{~L}$ & 32 & 0.998 \\
\hline
\end{tabular}


D. E. Gómez-Camargo et al.

\section{Continued}

\begin{tabular}{|c|c|c|c|c|c|c|c|c|c|c|c|c|c|c|c|c|c|c|}
\hline hc-364 & 15 & 14 & 30 & 24 & 11 & 13 & 13 & 14 & 12 & 12 & 19 & 15 & 17 & 23 & 12 & R1b & 57 & 1 \\
\hline hc-365 & - & 14 & 30 & 25 & 12 & 12 & 13 & 16 & - & 12 & 19 & 16 & 18 & 24 & 12 & R1b & 26 & 0.995 \\
\hline hc-373 & - & 14 & 30 & 25 & - & 14 & - & - & - & - & - & 16 & - & 23 & - & R1b & 44 & 0.794 \\
\hline hc-376 & - & 14 & - & 25 & 13 & 14 & - & 16 & - & - & - & 16 & 16 & 23 & - & R1b & 14 & 0.964 \\
\hline hc-377 & 13 & 14 & 30 & 25 & - & - & 12 & - & 13 & 13 & - & 16 & - & 23 & 12 & $\mathrm{R} 1 \mathrm{~b}$ & 20 & 0.613 \\
\hline hc-378 & - & 14 & 29 & 25 & 10 & 12 & - & 15 & - & - & - & 17 & 18 & 21 & - & I2a (xI2a1) & 29 & 0.411 \\
\hline hc-388 & 12 & 14 & 30 & 26 & 11 & 13 & 13 & 15 & - & - & - & 17 & 17 & 22 & 12 & $\mathrm{~L}$ & 27 & 0.778 \\
\hline hc-390 & - & 15 & 33 & 24 & 11 & 16 & - & 15 & - & - & - & 16 & 15 & 22 & - & R1b & 31 & 0.981 \\
\hline hc-391 & - & 14 & 33 & 24 & 11 & 16 & - & - & - & - & - & 16 & 15 & 22 & - & I2a1 & 34 & 0.864 \\
\hline hc-392 & - & 14 & 33 & 24 & 11 & 16 & - & 15 & - & - & - & 16 & 15 & 22 & - & $\mathrm{L}$ & 14 & 0.623 \\
\hline hc-393 & - & 14 & 33 & 24 & 11 & 16 & - & 15 & - & - & - & 16 & 15 & 22 & - & E1b1b & 25 & 0.503 \\
\hline hc-394 & 13 & 14 & 31 & 25 & 13 & 14 & 13 & 15 & 13 & - & - & 17 & 15 & 23 & 12 & $\mathrm{I} 2 \mathrm{~b} 1$ & 54 & 0.95 \\
\hline hc-395 & - & 13 & 30 & 23 & 11 & 12 & - & 17 & - & - & 21 & 16 & 17 & 20 & - & R1b & 33 & 0.843 \\
\hline hc-396 & 13 & 16 & 32 & 23 & 11 & 12 & 13 & 17 & 10 & 12 & 19 & 15 & 17 & 24 & 12 & $\mathrm{I} 2 \mathrm{~b} 1$ & 31 & 0.936 \\
\hline hc-398 & 14 & 15 & 31 & 23 & 11 & 12 & 13 & 15 & 10 & 11 & 20 & 16 & 17 & 22 & 11 & R1b & 33 & 1 \\
\hline hc-399 & - & 15 & - & 25 & 13 & 14 & - & 16 & - & - & - & 16 & 17 & 23 & - & $\mathrm{R} 1 \mathrm{~b}$ & 28 & 0.995 \\
\hline hc-402 & 12 & - & - & - & 11 & - & 13 & 15 & - & 12 & 19 & - & 15 & - & 11 & E1b1a & 32 & 0.771 \\
\hline hc-403 & - & 14 & 29 & 24 & - & 12 & - & - & - & - & - & 18 & - & 21 & - & $\mathrm{Q}$ & 20 & 0.687 \\
\hline hc-404 & - & 14 & 31 & 26 & 11 & 12 & - & 15 & - & 12 & - & 18 & 15 & 20 & - & I2b1 & 23 & 0.916 \\
\hline hc-405 & 13 & 14 & 29 & 24 & 12 & 14 & 12 & 16 & 13 & 12 & 18 & 16 & 18 & 24 & 12 & R1b & 18 & 0.879 \\
\hline hc-410 & - & 15 & 30 & 26 & 10 & 12 & - & 15 & - & - & - & 15 & 18 & 21 & - & R1b & 31 & 1,00 \\
\hline hc-412 & - & 14 & 30 & 25 & 11 & 12 & - & 15 & - & - & - & 17 & 15 & 23 & - & R1b & 44 & 0.993 \\
\hline hc-413 & - & 14 & 31 & 22 & 11 & 12 & - & 15 & - & - & - & 16 & 17 & 22 & - & J2a1 x J2a1-bh & 19 & 0.906 \\
\hline hc-414 & - & 14 & 30 & 25 & 11 & 12 & - & 15 & - & - & - & 16 & 19 & 21 & - & R1b & 31 & 0.991 \\
\hline hc-415 & - & 14 & 29 & 24 & 11 & 12 & - & 15 & - & 13 & - & 16 & 21 & 21 & - & E1b1b & 42 & 0.388 \\
\hline hc-416 & - & 15 & 29 & 24 & 12 & 14 & - & 16 & - & 12 & - & 16 & 17 & 23 & - & $\mathrm{R} 1 \mathrm{~b}$ & 14 & 1,00 \\
\hline hc-418 & - & 14 & 29 & 22 & 11 & 12 & - & 15 & - & - & - & 18 & 14 & 21 & - & $\mathrm{J} 2 \mathrm{~b}$ & 17 & 0.843 \\
\hline hc-419 & - & 14 & 29 & 25 & 12 & 14 & - & 16 & - & - & - & 17 & 18 & 23 & - & $\mathrm{R} 1 \mathrm{~b}$ & 30 & 0.979 \\
\hline hc-420 & - & 14 & 30 & 23 & 11 & 12 & - & 15 & - & 12 & - & 16 & 16 & 21 & - & J2a1 x J2a1-bh & 38 & 0.353 \\
\hline hc-421 & - & 14 & 31 & 24 & 11 & 12 & - & 16 & - & 11 & - & 16 & 14 & 20 & - & I2b1 & 47 & 0.462 \\
\hline hc-422 & 14 & 13 & 29 & 25 & 11 & 13 & 13 & 15 & 12 & 11 & 19 & 16 & 18 & 23 & 13 & $\mathrm{Q}$ & 35 & 0.983 \\
\hline hc-423 & 13 & 14 & 29 & 26 & 11 & 15 & 13 & 16 & 13 & - & - & 17 & 19 & 23 & 12 & R1b & 44 & 0.997 \\
\hline hc-424 & - & 15 & 31 & 24 & 11 & 14 & - & 15 & - & 12 & - & 18 & 16 & 21 & - & I2a (xI2a1) & 40 & 0.522 \\
\hline hc-425 & - & 13 & 29 & 25 & 11 & 12 & - & 15 & - & - & 20 & 16 & 18 & 21 & - & R1b & 58 & 1 \\
\hline hc-426 & - & - & - & - & 11 & 12 & 13 & 15 & - & 11 & 21 & - & 15 & - & - & I2b1 & 24 & 0.841 \\
\hline hc-427 & 16 & 13 & 30 & 23 & 11 & 13 & 12 & 18 & - & 11 & - & 16 & 14 & 20 & 11 & I2a (xI2a1) & 23 & 0.688 \\
\hline
\end{tabular}




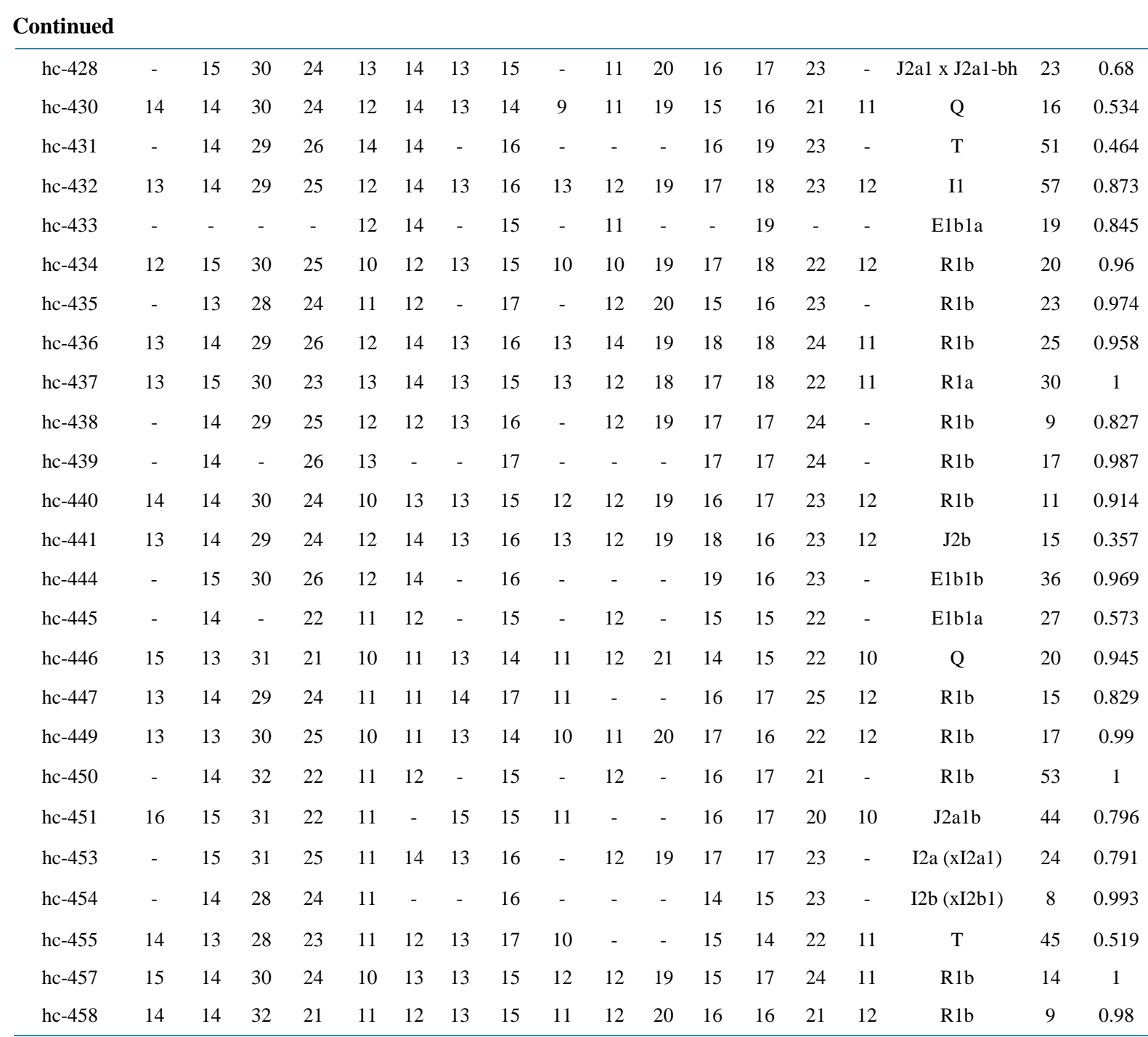

In order to know the haplotype distribution, locus diversity, and mean number of pairwise differences, we used only the complete haplotypes $(n=37)$. Out of 37 haplotypes studied, we found 36 different haplotypes suggesting high haplotype diversity. In addition, locus diversity over loci showed the highest values (1.000 \pm $0.0063)$, as well as the mean number of pairwise differences (10.084 \pm 4.7048$)$, whereas average gene diversity over loci obtained was $0.6722+0.3485$.

\subsection{Genetic Structure}

In order to know the genetic structure, we determined the frequency of haplogroups as well as fitness score and Bayesian probability using Haplogroup predictor software (Table 5). Our results showed that Cartagena de Indias was an admixture population represented by $\sim 80 \%$ European, $~ 10 \%$ Amerindian and $\sim 10 \%$ African. The most frequent haplogroups were R1b ( 40\%), I2a (xI2a1) (11\%) and Q ( 10\%), as well as E1b1a ( 5\%) and E1b1b ( 4\%). Additional haplogroups, evident in the low and moderate frequencies, were also found (G2a $\sim 1 \%$, I1 2\%, I2a1 3\%, I2b 2\%, I2b1 9\%, J2a1-bh 2\%, J2b 1\%, L 5\%, R1a, 1\% and T 5\%).

\subsection{Comparison with Other Populations}

We compared our data with previous results obtained in other Colombian populations. As can be seen in Figure 2, Cartagena's population maintains a genetic relationship with Antioquia, Magdalena and other populations 
Table 4. Allelic frequencies, descriptive statistical parameters and diversity index regarding the 15 STR loci of Cartagena de Indias population.

\begin{tabular}{|c|c|c|c|c|c|c|c|c|c|c|c|c|c|c|c|}
\hline 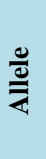 & $\begin{array}{l}\stackrel{\theta}{\omega} \\
\overrightarrow{0}\end{array}$ & 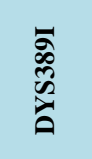 & 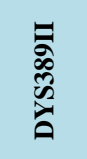 & 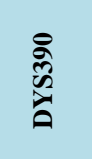 & $\begin{array}{l}\overline{\vec{\partial}} \\
\hat{\tilde{D}} \\
\vec{\theta}\end{array}$ & 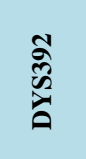 & 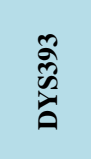 & 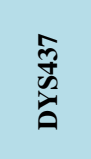 & 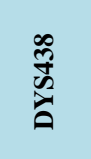 & 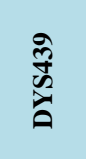 & $\begin{array}{l}\stackrel{゚}{Z} \\
\text { స્રે }\end{array}$ & 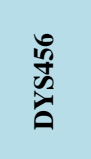 & 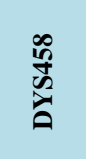 & 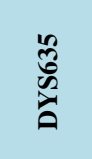 & 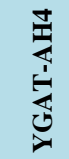 \\
\hline $\mathbf{n}$ & 66 & 122 & 111 & 123 & 114 & 102 & 78 & 124 & 60 & 91 & 79 & 123 & 123 & 123 & 68 \\
\hline 6 & - & - & - & - & - & - & - & - & - & - & - & - & - & - & - \\
\hline 7 & - & - & - & - & - & - & - & - & - & - & - & - & - & - & - \\
\hline 8 & - & - & - & - & - & - & - & - & 0.017 & - & - & - & - & - & - \\
\hline 9 & - & - & - & - & - & - & - & - & 0.083 & - & - & - & - & - & 0.015 \\
\hline 10 & - & - & - & - & 0.07 & 0.01 & - & - & 0.267 & 0.011 & - & - & - & - & 0.059 \\
\hline 11 & - & - & - & - & 0.64 & 0.02 & - & - & 0.233 & 0.297 & - & - & - & - & 0.338 \\
\hline 12 & 0.106 & - & - & - & 0.158 & 0.402 & 0.115 & - & 0.1 & 0.505 & - & - & - & - & 0.515 \\
\hline 13 & 0.394 & 0.205 & - & - & 0.123 & 0.098 & 0.756 & - & 0.3 & 0.176 & - & - & - & - & 0.074 \\
\hline 14 & 0.303 & 0.59 & - & - & 0.009 & 0.343 & 0.077 & 0.048 & - & 0.011 & - & 0.049 & 0.049 & - & - \\
\hline 15 & 0.136 & 0.189 & - & - & - & 0.088 & 0.051 & 0.508 & - & - & - & 0.154 & 0.203 & - & - \\
\hline 16 & 0.045 & 0.016 & - & - & - & 0.039 & - & 0.323 & - & - & - & 0.455 & 0.195 & - & - \\
\hline 17 & 0.015 & - & - & - & - & - & - & 0.097 & - & - & - & 0.244 & 0.35 & - & - \\
\hline 18 & - & - & - & - & - & - & - & 0.024 & - & - & 0.063 & 0.081 & 0.13 & - & - \\
\hline 19 & - & - & - & - & - & - & - & - & - & - & 0.481 & 0.016 & 0.065 & 0.008 & - \\
\hline 20 & - & - & - & - & - & - & - & - & - & - & 0.253 & - & - & 0.041 & - \\
\hline 21 & - & - & - & 0.024 & - & - & - & - & - & - & 0.177 & - & 0.008 & 0.26 & - \\
\hline 22 & - & - & - & 0.106 & - & - & - & - & - & - & 0.025 & - & - & 0.171 & - \\
\hline 23 & - & - & - & 0.138 & - & - & - & - & - & - & - & - & - & 0.382 & - \\
\hline 24 & - & - & - & 0.285 & - & - & - & - & - & - & - & - & - & 0.122 & - \\
\hline 25 & - & - & - & 0.333 & - & - & - & - & - & - & - & - & - & 0.016 & - \\
\hline 26 & - & - & - & 0.106 & - & - & - & - & - & - & - & - & - & - & - \\
\hline 27 & - & - & - & - & - & - & - & - & - & - & - & - & - & - & - \\
\hline 28 & - & - & 0.108 & - & - & - & - & - & - & - & - & - & - & - & - \\
\hline 29 & - & - & 0.342 & 0.008 & - & - & - & - & - & - & - & - & - & - & - \\
\hline 30 & - & - & 0.324 & - & - & - & - & - & - & - & - & - & - & - & - \\
\hline 31 & - & - & 0.144 & - & - & - & - & - & - & - & - & - & - & - & - \\
\hline 32 & - & - & 0.045 & - & - & - & - & - & - & - & - & - & - & - & - \\
\hline 33 & - & - & 0.036 & - & - & - & - & - & - & - & - & - & - & - & - \\
\hline$k$ & 6 & 4 & 6 & 7 & 5 & 7 & 4 & 5 & 6 & 5 & 5 & 6 & 7 & 7 & 5 \\
\hline$h$ & 0.721 & 0.574 & 0.742 & 0.766 & 0.545 & 0.701 & 0.406 & 0.626 & 0.767 & 0.625 & 0.668 & 0.7 & 0.775 & 0.74 & 0.612 \\
\hline
\end{tabular}

$\mathrm{n}$ : number of individuals studied; $k$ : number of alleles; $h$ : locus diversity. 
Table 5. Cartagena de Indias haplogroup frequencies.

\begin{tabular}{cc}
\hline Haplogroup & Frequency \\
\hline E1b1a & 0.045 \\
E1b1b & 0.038 \\
G2a & 0.007 \\
I1 & 0.015 \\
I2a (xI2a1) & 0.114 \\
I2a1 & 0.03 \\
I2b (xI2b1) & 0.015 \\
I2b1 & 0.091 \\
J2a1b & 0.015 \\
J2a1 × J2a1-bh & 0.022 \\
J2b & 0.007 \\
L & 0.045 \\
Q & 0.099 \\
R1a & 0.007 \\
R1b & 0.381 \\
T & 0.053 \\
\hline
\end{tabular}

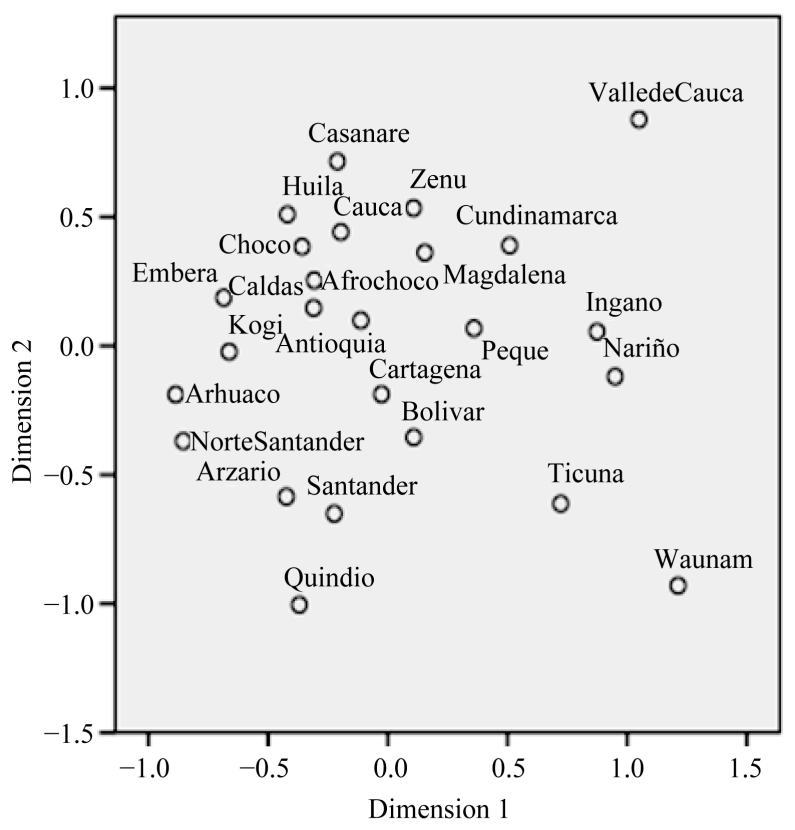

Figure 2. MDS plot of Colombian populations RsT pairwise differences using 15 Y-STR loci.

from the Department of Bolivar. Moreover, Cartagena population is in the centre of the Colombian populations with different ancestries, underlining the complexity of this population.

\subsection{Network Analysis}

In order to establish the genetic relationship within each lineage, a median joining network was constructed 
(Figure 3). The R1b haplogroup shows a star-like network, indicating that Cartagena's population is closely related to the Western European populations of Majorca and Valencia (Iberian Peninsula). In addition, a separate group of R1b Cartagena men related to Sicily's population, suggests a high genetic diversity even within this lineage (Figure 3(a)). Moreover, the I2a (xI2a1) lineage shows a star-like network suggesting that Cartagena's population could be related to a young population that may have suffered some demographic events (e.g. bottleneck, genetic drift, and founder effects). With respect to the Q haplogroup, we compared our data with Q-M242

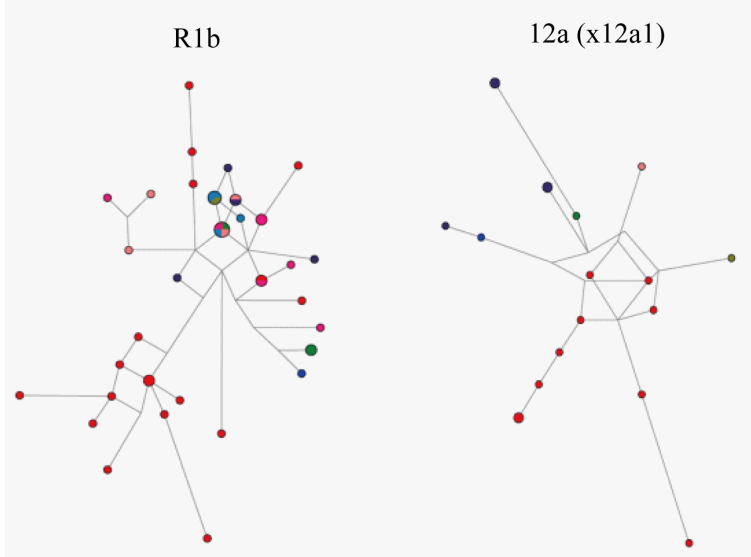

(a)

E1bla

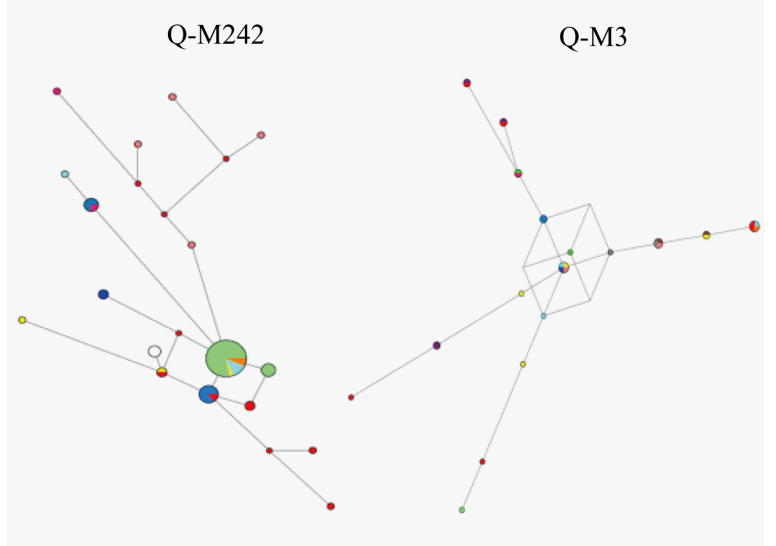

(b)

E1blb

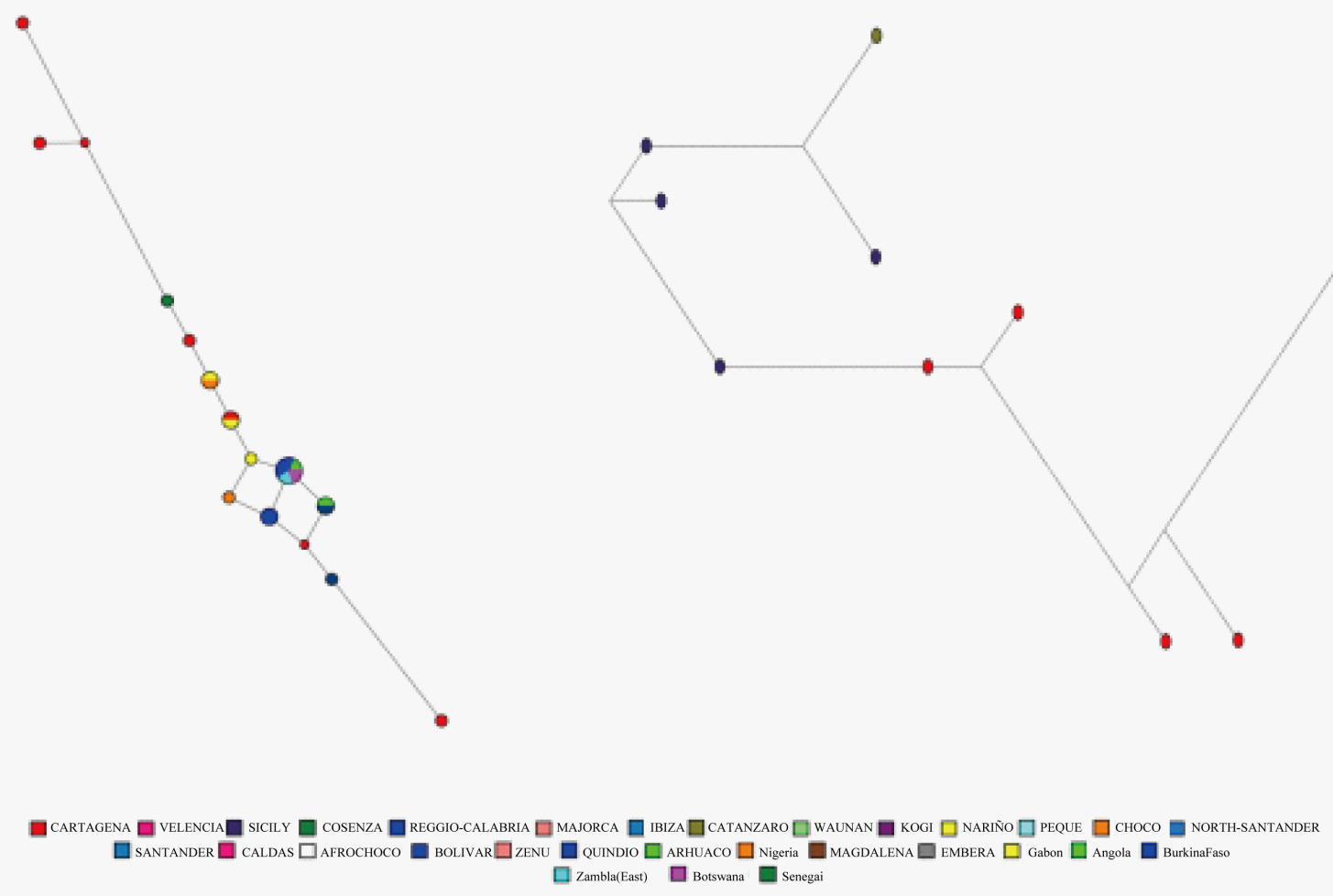

(c)

Figure 3. Median joining network of ancestral lineages in Cartagena de Indias population. (a) European lineages: R1b and I2a (xI2a1); (b) Amerindian lineages: Q-M242 and Q-M3; (c) African lineages: E1b1a and E1b1b. 
and Q-M3 haplogroups described previously [3]. Cartagena's population showed a lineage closely related with Waunan (Q-M242) and Zenú ethnic groups. In addition, our haplogroups Q are related to Amerindian populations such as Kogi and Arhuacos (Figure 3(b)). Nevertheless, this diversity of pattern shows lineages poorly characterized by the lack of availability of markers that allow a higher resolution. Our results also point to the important African ancestry in Cartagena's population represented by E1b1a and E1b1b lineages (Figure 3(c)). Interestingly, Cartagena's population also has a patrilineal relationship with Senegal and Gabon.

\section{Discussion}

Population stratification is one of the most important confounding factors in population-based genetic association studies, provoking $40 \%$ of spurious associations [20]. These false associations are more frequent in Latino populations, heterogeneous populations in which a dissimilar ancestry proportion give rise to each subpopulation not being equally represented [21]-[24]. Consequently, in recent years much research has focused on detecting the population stratification before beginning genetic association studies of complex diseases in order to avoid spurious associations [5] [25] [26].

The contemporary Cartagena de Indias population emerged from recent miscegenation (500 years ago) as a cosmopolitan city where Spanish conquerors mixed with Native American people derived principally from Karib, Malibu, Arawak and Chibcha language families [27]. As stated above, this admixture was asymmetrical and it is in agreement with our findings which show that the Cartagena sample studied is comprised of $\sim 80 \%$ European, $\sim 10 \%$ Amerindian and $\sim 10 \%$ African ancestries. With respect to the European ancestry, it was principally represented by the haplogroup R1b (hg-R1b), which was present in $~ 50 \%$ of the total European haplogroups found in Cartagena's population. The hg-R1b is the result of the admixture with the Spanish conquerors during the colonial period, because Cartagena was one of the most important Spanish settlements in America [28] [29]. This haplogroup is actually present in more than $60 \%$ of the Spaniard population [30], as well as $\sim 80 \%$ of Basque Country population [17] [31]. However, hg-R1b could be also related to Mediterranean populations [17], since it showed a haplotype relationship with Cartagena's population (Figure 3(a)). In addition, the Italian population could also participate in introducing the other important European lineage (haplogroup I2a (xI2a1)). This haplogroup is one of the most frequent in the island of Sardinia as well as the Mediterranean region [15], and could be related to the Italian migrations 200 years ago [28]. These Italian migrations came from Sicily and Cosenza principally, and settled down on the northern coast of Colombia (Barranquilla, Cartagena and Santa Marta) [32]. With respect to the other European lineages, these could be related to pirates and corsairs from England, France, Portugal and the Netherlands, who continuously invaded Cartagena de Indias because this city was the principal port for gold and silver during the colonial period [29] [33].

Apart from the European ancestry, Cartagena showed an important contribution from African lineages, which were represented by the haplogroups E1b1a and E1b1b. As mentioned before, thousands of African slaves especially those from Western and Central Africa were introduced in the 16th century [2], which disembarked on the Pacific and Atlantic Coasts [34]. The African lineages increased noticeably the diversity of Cartagena's population because they represented different clans from Senegambia, Ivory Coast, Central Africa, Congo, Angola and Mozambique among others; many of them were found in Cartagena's population [3] [35] (Figure 3).

Both ancestries (European and African) were admixed with native Amerindian populations, which actually maintained an ancestral relationship with Cartagena's population. On the other hand, our results suggested that the Amerindian diversity of Cartagena was related with Waunan, Kogui, Chocó, Pequé and Zenú groups, all of which were related to the Q-M242/Q-M3 lineages, which represented the majority of Amerindian Y chromosomes [36]. Nevertheless, the Amerindian diversity of Cartagena's population could show even more heterogeneity, which could be related to the diversity inside the Q haplogroup [3].

The great genetic diversity of Cartagena de Indias' population, represented by mestizo, Afro-Colombian, and Amerindian lineages, supports the importance of ancestral studies in admixture populations. Our results suggest an important substructure degree within Cartagena's population. This dissimilar ancestral proportion indicates the necessity to increase the resolution as well as the use of different genetic markers in order to elucidate the complex population history of Cartagena.

Although different research groups have also studied Colombian and Cartagena samples populations, the regional differences, the demographic events, and the complex patterns of diversity suggest examining different samples of the same population in order to represent the whole genetic complexity [5] [37]-[39]. Nevertheless, 
these ancestral patterns should not be applied to the entire Colombian population, because the demographic events and consequently the diversity patterns are specific of each population.

In addition, our results emphasize the contribution of population genetics in population-based genetic association studies, where the ethnic self-identification is not appropriate to correct the population stratification. Our data could contribute to avoid or diminish statistical errors type 1 and 2, which is a fundamental strategy in the search of disease biomarkers.

\section{Acknowledgements}

This study was partially supported by the Kellogg’s Nutritional and Health Institute-Mexico (to R.G.), Departamento Administrativo de Ciencia, Tecnología e Inovación, University of Cartagena, Colombia (grant $\mathrm{N}^{\circ}$ 110765741638), as well as National University from Colombia. We also thank all Cartagena people for their enthusiastic participation for whose collaboration made this study possible, as well as Laboratorio de Genómica, Proteómica y Metabolómica from LaNSE-Cinvestav-México for helping in Genotyping processes.

\section{References}

[1] Lemaitre, E., Herazo, D.B. and Patrón, F.S. (1983) Historia General De Cartagena. Banco de la Republica, Bogotá.

[2] Wheat, D. (2011) The First Great Waves: African Provenance Zones for the Transatlantic Slave Trade to Cartagena De Indias, 1570-1640. The Journal of African History, 52, 1-22. http://dx.doi.org/10.1017/S0021853711000119

[3] Rojas, W., Parra, M.V., Campo, O., Caro, M.A., Lopera, J.G., Arias, W., et al.(2010) Genetic Make up and Structure of Colombian Populations by Means of Uniparental and Biparental DNA Markers. American Journal of Physical Anthropology, 143, 13-20. http://dx.doi.org/10.1002/ajpa.21270

[4] Bryc, K., Velez, C., Karafet, T., Moreno-Estrada, A., Reynolds, A., Auton, A., et al. (2010) Colloquium Paper: GenomeWide Patterns of Population Structure and Admixture among Hispanic/Latino Populations. Proceedings of the National Academy of Sciences of the United States of America , 107, 8954-8961. http://dx.doi.org/10.1073/pnas.0914618107

[5] Vergara, C., Caraballo, L., Mercado, D., Jimenez, S., Rojas, W., Rafaels, N., et al. (2009) African Ancestry Is Associated with Risk of Asthma and High Total Serum IgE in a Population from the Caribbean Coast of Colombia. Human Genetics, 125, 565-579. http://dx.doi.org/10.1007/s00439-009-0649-2

[6] Attia, J., Ioannidis, J.P., Thakkinstian, A., McEvoy, M., Scott, R.J., Minelli, C., et al. (2009) How to Use an Article About Genetic Association: B: Are the Results of the Study Valid? JAMA: The Journal of the American Medical Association, 301, 191-197. http://dx.doi.org/10.1001/jama.2008.946

[7] Bouaziz, M., Paccard, C., Guedj, M. and Ambroise, C. (2012) Ships: Spectral Hierarchical Clustering for the Inference of Population Structure in Genetic Studies. PLoS One, 7,e45685. http://dx.doi.org/10.1371/journal.pone.0045685

[8] Neuditschko, M., Khatkar, M.S. and Raadsma, H.W. (2012) Netview: A High-Definition Network-Visualization Approach to Detect Fine-Scale Population Structures from Genome-Wide Patterns of Variation. PLoS One, 7, e48375. http://dx.doi.org/10.1371/journal.pone.0048375

[9] Wang, S.J., Ray, N., Rojas, W., Parra, M.V., Bedoya, G., Gallo, C., et al. (2008) Geographic Patterns of Genome Admixture in Latin American Mestizos. PLoS Genetics, 4, e1000037. http://dx.doi.org/10.1371/journal.pgen.1000037

[10] Little, J., Higgins, J.P., Ioannidis, J.P., Moher, D., Gagnon, F., von Elm, E., et al. (2009) Strengthening the Reporting of Genetic Association Studies (STREGA) - an Extension of the STROBE Statement. European Journal of Clinical Investigation, 39, 247-266. http://dx.doi.org/10.1111/j.1365-2362.2009.02125.x

[11] Butler, J.M., Schoske, R., Vallone, P.M., Kline, M.C., Redd, A.J. and Hammer, M.F. (2002) A Novel Multiplex for Simultaneous Amplification of 20 Y Chromosome STR Markers. Forensic Science International, 129, 10-24. http://dx.doi.org/10.1016/S0379-0738(02)00195-0

[12] Butler, J.M., Schoske, R., Vallone, P.M., Redman, J.W. and Kline, M.C. (2003) Allele Frequencies for 15 Autosomal STR Loci on U.S. Caucasian, African American, and Hispanic Populations. Journal of Forensic Sciences, 48, 908-911.

[13] Magana, J.J., Gomez, R., Maldonado-Rodriguez, M., Velazquez-Perez, L., Tapia-Guerrero, Y.S., Cortes, H., et al. (2013) Origin of the Spinocerebellar Ataxia Type 7 Gene Mutation in Mexican Population. The Cerebellum, 12, 902-905. http://dx.doi.org/10.1007/s12311-013-0505-8

[14] Excoffie, L. and Lischer, H.E.L. (2010) Arlequin Suite Ver 3.5: A New Series of Programs to Perform Population Genetic Analyses under Linux and Windows. Molecular Ecology Resources, 10, 564-567. http://dx.doi.org/10.1111/j.1755-0998.2010.02847.x

[15] Yunis, J.J., Acevedo, L.E., Campo, D.S. and Yunis, E.J. (2005) Population Data of Y-STR Minimal Haplotypes in a Sample of Caucasian-Mestizo and African Descent Individuals of Colombia. Forensic Science International, 151, 307-313. 
http://dx.doi.org/10.1016/j.forsciint.2005.02.005

[16] de Filippo, C., Barbieri, C., Whitten, M., Mpoloka, S.W., Gunnarsdottir, E.D., Bostoen, K., et al. (2011) Y-Chromosomal Variation in Sub-Saharan Africa: Insights into the History of Niger-Congo Groups. Molecular Biology and Evolution, 28, 1255-1269. http://dx.doi.org/10.1093/molbev/msq312

[17] Rodriguez, V., Tomas, C., Sanchez, J.J., Castro, J.A., Ramon, M.M., Barbaro, A., et al. (2009) Genetic Sub-Structure in Western Mediterranean Populations Revealed by 12 Y-Chromosome STR Loci. International Journal of Legal Medicine, 123, 137-141. http://dx.doi.org/10.1007/s00414-008-0302-y

[18] Athey, T.W. (2005) Haplogroup Prediction from Y-STR Values Using an Allele-Frequency Approach. Journal of Genetic Genealogy, 1, 1-7.

[19] Bandelt, H.J., Forster, P. and Rohl, A. (1999) Median-Joining Networks for Inferring Intraspecific Phylogenies. Molecular Biology and Evolution, 16, 37-48. http://dx.doi.org/10.1093/oxfordjournals.molbev.a026036

[20] O’Connor, T.D., Kiezun, A., Bamshad, M., Rich, S.S., Smith, J.D., Turner, E., et al. (2013) Fine-Scale Patterns of Population Stratification Confound Rare Variant Association Tests. PLOS ONE, 8, e65834. http://dx.doi.org/10.1371/journal.pone.0065834

[21] Tsai, H.J., Choudhry, S., Naqvi, M., Rodriguez-Cintron, W., Burchard, E.G. and Ziv, E. (2005) Comparison of Three Methods to Estimate Genetic Ancestry and Control for Stratification in Genetic Association Studies among Admixed Populations. Human Genetics, 118, 424-433. http://dx.doi.org/10.1007/s00439-005-0067-z

[22] Choudhry, S., Coyle, N.E., Tang, H., Salari, K., Lind, D., Clark, S.L., et al. (2006) Population Stratification Confounds Genetic Association Studies among Latinos. Human Genetics, 118, 652-664. http://dx.doi.org/10.1007/s00439-005-0071-3

[23] Hao, K., Chudin, E., Greenawalt, D. and Schadt, E.E. (2010) Magnitude of Stratification in Human Populations and Impacts on Genome Wide Association Studies. PLOS ONE, 5, e8695. http://dx.doi.org/10.1371/journal.pone.0008695

[24] Jiang, Y., Epstein, M.P. and Conneely, K.N. (2013) Assessing the Impact of Population Stratification on Association Studies of Rare Variation. Human Heredity, 76, 28-35. http://dx.doi.org/10.1159/000353270

[25] Abdellaoui, A., Hottenga, J.J., Xiao, X., Scheet, P., Ehli, E.A., Davies, G.E., et al. (2013) Association between Autozygosity and Major Depression: Stratification Due to Religious Assortment. Behavior Genetics, 43, 455-467.

[26] Nievergelt, C.M., Maihofer, A.X., Shekhtman, T., Libiger, O., Wang, X., Kidd, K.K., et al. (2013) Inference of Human Continental Origin and Admixture Proportions Using a Highly Discriminative Ancestry Informative 41-SNP Panel. Investigative Genetics, 4, 13-19.

[27] Lleras Perez, R. (1993) La Historia Prehispánica Tardía Del Noroeste De Suramérica 800 a 1500 D.C. Revista de Arqueología Americana, 91, 51-70.

[28] Aguilera Diaz, M. and Meisel Roca, A. (2009) Tres Siglos De Historia Demográfica De Cartagena De Indias. Banco de la República, Bogotá.

[29] Múnera, A. and Molina, L.F. (1998) El Fracaso De La Nación: Región, Clase Y Raza En El Caribe Colombiano (17171821). El Ancora Editores, Banco de la República.

[30] Adams, S.M., Bosch, E., Balaresque, P.L., Ballereau, S.J., Lee, A.C., Arroyo, E., et al. (2008) The Genetic Legacy of Religious Diversity and Intolerance: Paternal Lineages of Christians, Jews, and Muslims in the Iberian Peninsula. American Journal of Human Genetics, 83, 725-736. http://dx.doi.org/10.1016/j.ajhg.2008.11.007

[31] Young, K.L., Sun, G., Deka, R. and Crawford, M.H. (2011) Paternal Genetic History of the Basque Population of Spain. Human Biology, 83, 455-475. http://dx.doi.org/10.3378/027.083.0402

[32] Capelli, V. (2006) Entre “Macondo” Y Barranquilla. Los Italianos En La Colombia Caribea. De Finales Del Siglo XIX Hasta La Segunda Guerra Mundial. Memoria \& Sociedad, 10, 25-48.

[33] Lasso, M. (2006) Race War and Nation in Caribbean Gran Colombia, Cartagena, 1810-1832. The American Historical Review, 111, 336-361. http://dx.doi.org/10.1086/ahr.111.2.336

[34] Rodas, C., Gelvez, N. and Keyeux, G. (2003) Mitochondrial DNA Studies Show Asymmetrical Amerindian Admixture in Afro-Colombian and Mestizo Populations. Human Biology, 75, 13-30. http://dx.doi.org/10.1353/hub.2003.0026

[35] Friedemann, N. (2000) Huellas De Africanía En La Diversidad Colombiana. In: Encuentros De Africanía, F.C.C. Negra, Bogotá.

[36] Schurr, T.G. and Sherry, S.T. (2004) Mitochondrial DNA and Y Chromosome Diversity and the Peopling of the Americas: Evolutionary and Demographic Evidence. American Journal of Human Biology, 16, 420-439. http://dx.doi.org/10.1002/ajhb.20041

[37] Benn-Torres, J., Bonilla, C., Robbins, C.M., Waterman, L., Moses, T.Y., Hernandez, W., et al. (2008) Admixture and Population Stratification in African Caribbean Populations. Annals of Human Genetics, 72, 90-98. 
[38] Mesa, N.R., Mondragon, M.C., Soto, I.D., Parra, M.V., Duque, C., Ortiz-Barrientos, D., et al. (2000) Autosomal, mtDNA, and Y-Chromosome Diversity in Amerinds: Pre- and Post-Columbian Patterns of Gene Flow in South America. American Journal of Human Genetics, 67, 1277-1286.

[39] Bedoya, G., Montoya, P., Garcia, J., Soto, I., Bourgeois, S., Carvajal, L., et al. (2006) Admixture Dynamics in Hispanics: A Shift in the Nuclear Genetic Ancestry of a South American Population Isolate. Proceedings of the National Academy of Sciences of the United States of America, 103, 7234-7239. http://dx.doi.org/10.1073/pnas.0508716103

[40] Gusmão, L., Butler, J.M., Carracedo, A., Gill, P., Kayser, M., Mayr, W.R., et al. (2006) DNA Commission of the International Society of Forensic Genetics (ISFG): An Update of the Recommendations on the Use of Y-STRs in Forensic Analysis. Forensic Science International, 157, 187-197. http://dx.doi.org/10.1016/j.forsciint.2005.04.002

[41] Redd, A.J., Agellon, A.B., Kearney, V.A., Contreras, V.A., Karafet, T., Park, H., et al. (2002) Forensic Value of 14 Novel STRs on the Human Y Chromosome. Forensic Science International, 130, 97-111. http://dx.doi.org/10.1016/S0379-0738(02)00347-X

[42] White, P.S., Tatum, O.L., Deaven, L.L. and Longmire, J.L. (1999) New, Male-Specific Microsatellite Markers from the Human Y Chromosome. Genomics, 57, 433-437. http://dx.doi.org/10.1006/geno.1999.5782 